\title{
Gay Lingo as Reflection of Social Identity
}

\author{
Ramea Maestrecampo Romero \\ Asst. Professor II, Faculty of Teacher Development \\ Philippine Normal University Visayas \\ \{romero.rm@pnu.edu.ph\}
}

\begin{abstract}
Gay Lingo as Reflection of Social Identity" explores the use of gay lingos as word substitutes in conversations. Frameworks were based on Social Identity Theory [ 4 ], Sociolinguistics by [ 1 ],Queer Theory [ 5 ], and Sociolinguistics of [ 6 ]. Methods used were Descriptive-qualitative design and quantitative approach. Data were based on group discussions and interviews. Findings identified the functions of gay lingo, circumstances that allow Filipino teenagers to use gay lingos, and Social Identity projection. This study recommends that language instructors in a college/university must see the strengths and weaknesses of self-confessed closeted gay men yet creative individuals.
\end{abstract}

Keywords: Gay Lingo, Reflection, Social Identity

\section{INTRODUCTION}

People use a language whenever they interact with others in a society. Without a language, there will be trouble. No people or no society survived without a language.

Over the past twenty years, sociolinguists have developed increasingly sophisticated ways of modelling the relationship between language, and social identity and have attracted various studies by researchers, linguists, and educators in language and gender. Generally speaking, most of the studies on language, gender, and social identity focus on the aspect of gender differences in language.

As [ 1 ] states, "A major topic in sociolinguistics is the connection, if any, between the structures, vocabularies, and ways of using particular languages and the social roles of the men and women who speak these languages".

Filipino language is dynamic since it does not conform itself on a single culture but allows more freedom to its speakers. People love to use colorful words to hide real meanings and to remain more distinct from other gender minority groups for common understanding of the language. One of these languages is invented by gays which remains distinct because they have their own shifting code called Gay Lingo. It is original and creative in a manner even if it is from a local word which is spoken with gusto, given a twist, peculiar and defined cleverly by gay

This study examined what happens in the conversations of the self-confessed gay Filipino teenagers. Due to wide acceptance of this language, the gay lingo is used by both male and female in their daily conversations. In the community where they belong, the gay lingos may seem to function as a reflection of their social identity.

The main purpose of this study was to identify modern Filipino gay lingo word substitutes in conversation among self-confessed gay students. Specifically, this study sought to answer 
the following questions:1. What are the common gay lingos used as word substitute by participants during conversation? 2. How do identified gay lingos function?3. Under what circumstances do the participants often use gay lingos as wordsubstitute?3.1. When do gay students use the word substitute?3.2. Where do gay students use the word substitute?3.3. Why do gay students use the word substitute?3.4. How do gay students use the word substitute?4. How does gay lingo project gay students' social identity?

This study is anchored on the different theories related to language acquisition such as Social Identity [ 4 ], Sociolinguistics by [ 1 ], Queer Theory by [5 ], and Sociolinguistics of [ $6]$.

On Social Identity , [ 1 ] refer Social Identity Theory to an individual's sense of belonging to a group. Furthermore, social identity has been cited as one of the most relevant constructs when understanding inter-group relations.According to [ 7 ], social identity is often referred to as collective identity or group identity.

According to [ 1 ], the word sociolinguistics contains two words. First is "society" which means any group of people who are drawn together for certain purpose; and, the second is "language" which means what the members of particular society speak. Sociolinguistics is the study of kinds of language used is social context. There are so many phenomena to the function of language than can be found in our society. The common phenomenon than can be found is code switching. This phenomenon exists because there is the social context which allows people who live in the communities to communicate in more than one language.

According to [ 5 ], Queer theory is not a singular or systematic conceptual or methodological framework, but a collection of intellectual engagements with the relations between sex, gender and sexual desire. If queer theory is a school of thought, then it's one with a highly unorthodox view of discipline. The term describes a diverse range of critical practices and priorities: readings of the representation of same-sex desire in literary texts, films, music, images; analyses of the social and political power relations of sexuality; critiques of the sex-gender system; studies of transsexual and transgender identification, of sadomasochism and of desires.

According to [ 6 ], "sociolinguistics is the study of language and society".This study was anchored on the different concepts related to gay terms used by the participants during conversation.

\section{a. Functions of Gay Lingo}

Language serves different and special functions. It serves to express social and personal relations, to represent an experience, to impart factual information, to control the behavior of others, to get involved in fantasies, and to show one's identity .

\section{b. Gay Lingo as Used in Filipino Context}

[11] stressed that Filipino gays used wonderful language, gay lingo. In general seamlessly switch into when they are gathered together or most immediately when they are around other people in order perhaps to "cloak" their intimate conversations, the better to protect the "virgin" ears.

[14] stressed that to Filipino speakers, Sward Speak sounds witty and twangy, and it immediately identifies the speaker as homosexual Gay lingo has been prevalent throughout the Philippines, and was initially used by the homosexual community as a tool to communicate with each other. 


\section{c. Studies on Gay Lingos in Filipino Context}

[ 12 ] mentioned in his study that the gay language in the Philippines is a 'pidgin'. Filipinos of the third sex decided to come up with a form of language that is only peculiar to them to facilitate communication among the members with a slight intention of concealing the real message behind the information being expressed.

[15] pointed out the need to have a Computer Pidgin Language (CPL) purposely to teach people a new language that is efficient for dialogues with computers. Their study is inspired by the aim to create a completely new language that people would have to learn and practice to facilitate easy operation of the gadget.' For some consideration, it is learned that the words and terms used in this "Philippine gay language" quickly undergo changes due to the speed contribution shared by the "speakers" who come from the different places in the country

\section{METHODOLOGY}

a) Research Design

The researcher employed the descriptive-qualitative design; more specifically, the sequential explanatory design which is characterized by the collection and analysis of descriptive data followed by the collection and analysis of qualitative data [18]. In the descriptive phase, the researcher used a survey instrument to identify and classify the gay lingos used by gays as word substitutes for conversations.

In terms of qualitative approach, the data used for this study was collected through focused group discussions and interviews.

The researcher used a Focused Group Discussion (FGD) to expose students' reasoning on the question "under what circumstances do Filipino teenagers often use gay lingos as word.The data collected were transcribed in order to form interpretations. In order to answer specific objectives 2, 3 and 4, the researcher did a Focused Group Discussion involving eight participants who accepted the invitation for a recorded conversation.

Categorization of themes was done to illustrate the functions of gay lingos, the circumstances that allow Filipino teenagers to often use the gay lingos as word substitute and on how gay lingos project Social Identity.

In terms of quantitative approach, profiling of the participants and frequency count of common gay lingos used as substitute words were utilized. In this study, top ten commonly gay lingos were ranked as word substitutes during conversations.

In terms of Thematic Analysis, The data collected through interviews were analysed in a similar way based on a three-stage procedure. First, the familiarisation with data was internalised through transcription of the interviews. The audio recordings of the interviews of eight participants were listened to a number of times for accurate transcription. Second, the transcripts and audio recordings were coded on it. The third stage was the theme development. Using the thematic analysis of interview data served the purpose of this study as it enabled the effective categorization.

\section{b) Participants}

This study involved 20 self-confessed gay students who are currently enrolled in one of the state universities in the north. Some of them are overt gays and self-confessed users of gay lingo in their everyday conversations with members of their "straight" classmates and friends. Purposive sampling was employed by the researcher due to the characteristics of the population and nature of the study. [21] suggested that purposive sampling is preferred for four specific reasons. Two of the reasons include: their being self-confessed gays and their 
being users of gay lingos. For qualitative results, participants were randomly chosen for FGD. There were a total of 8 participants for the said FGD.

\section{c) Instruments}

The research instrument used in this study was of two types. The first was a pre-survey instrument used to identify gay lingos used by gays as substitute for conversation. This was an open ended questionnaire from which students enumerated the gay terms they used during their conversation. The second part of the research instrument was of two parts. Part One dealt with the personal data of the participants. This included participants' name (optional), type of community, language spoken at home, and the language source. Part Two of the research instrument was a language check. In this part of the questionnaire, 45 gay terms were included based from the pre-survey conducted. Participants were asked to put a meaning in each of the term in the second column while the third column was for those gay terms where they were not familiar with.

d) Data

The data used in the study are the generated common gay lingos, recorded FGD transcripts and their translations.

e) Data Gathering Procedure

1. Permission

Before the conduct of the study, the researcher provided permission letter for the Dean for Academics of place of the conduct of the study for approval, and then the study observed proper procedure.

\section{Face-to-face encounter/ Administration of Survey Questionnaire}

Face-to face encounter was conducted and the participants were asked to provide necessary information from the self-administered questionnaire. The identified participants answered the questionnaire during their vacant period while hanging out in their "Tambayan" (canteen, covered court, benches area). Participants read the items carefully. They wrote the meanings of the gay lingo words in the appropriate column. If the word was not familiar to them, they put a $(\sqrt{ })$ under the appropriate column.

\section{Retrieval of Instruments}

Survey instruments were retrieved by the researcher after the face -to-face encounter with the participants and analyzed them together with inter-coders and inter raters then placed these results on tables.

\section{Collation of Results}

The researcher, with the help of inter-coders, needed to manually transfer the responses from the questionnaires into a spread sheet or a table and put each question number as a column heading, and use one row for each person's answers. They then assigned each possible answer a number or 'code' then went through each participant's questionnaire in turn, adding in the codes then entered this data into a spreadsheet or table. Checking of some of the data for accuracy was done after 


\section{Focus Group Discussion}

Focused group discussion was guided by the researcher (or group facilitator) who introduced topics for discussion and helped the group to participate in a lively and natural discussion among them.

To get data for unanswered items or for clarity of entries in the questionnaire, the researcher immediately conducted informal conversations with the participants. The information derived from this was considered as responses for problem numbers 2,3 and 4 .

\section{f) Validity and Reliability}

To establish the validity and reliability of the instruments on the commonly used word substitute and recorded conversations of self-confessed gay participants, a total of three intercoders, and three inter-raters were asked to validate and evaluate the study. Since the scope of the was quite huge, the researcher thought of having inter-coders so as to distribute the volumes of work and have analysed outputs ready in time. Thirty percent (30\%) of the data purposively selected from the data were inter-coded by the inter-coders who happened to be college faculty and are experts in their field of specialization. A discussion related to the concepts and frameworks was facilitated by the researcher. When the same understanding and interpretation of those concepts had been agreed, the researcher and the inter-coders proceeded to analyse and gave judgement independently. After completing the rating and coding work, both the researcher and the inter-coders compared the results and both arrived at the high level of agreement. Some differences in the results were observed, discussed, reviewed and recorded until the final agreement.

\section{g) Ethical Consideration}

For ethical consideration and to ensure privacy and confidentiality, an informed consent form to each participant during FGD interview, and assurance/reminders that no one would be charged for their participation in this study. Participants were informed that their participations would not affect any of their engagements in school and in the community.

When they agreed to participate in this FGD, they were asked to sign over their names to signify their intentions. The names would not be used in any report, but the comments and suggestions would help researcher achieve the objectives of this study. The data and their transcriptions would be kept for two years and be discarded thereafter.

\section{FINDINGS AND DISCUSSION}

This chapter includes the presentation, analysis and interpretation of the results of the study with regard to the commonly used gay lingos as word substitutes, the thematic analysis on the identified functions of gay lingos, circumstances that may allow Filipino teenagers to often use gay lingos as word substitutes, and how gay lingo projects social identity.Table 1 reflects the common gay lingos used as word substitutes during conversation. This shows that $95 \%$ of the participants commonly used the word "chaka", $90 \%$ of the participants commonly used the word "itich", $85 \%$ of the participants commonly used the words "Wiz"/"Washington", "Charing"/“tienes", "rampa"/"rampage", and "Kever ko", and 80\% of them commonly used the words "backstreet boys", "award", "emote", and "jutay"/"jutes". The word "chaka" ranked first from the top 10 common gay lingos, the word "Itich" ranked second, the words "Wiz"/"Washington",

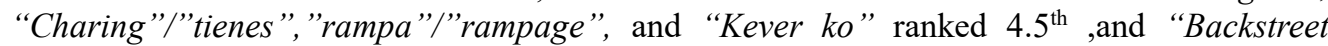
boys" "Award", "Emote" and "Jutay"/"jutes" ranked 7.5 . 
The implications of the use of these gay lingos show the gays' ways of integrating themselves in the society in their own terms, making serious as satirized or light and makes the language as an act of subversion.

\section{Qualitative Presentation}

The researcher came to know the participants through interviews, journeying with them and listening to their experiences. The researcher categorized their narrative responses and descriptions as follows: On Functions of Gay Lingo (Thematic Insights), in the context of the present study, as mentioned by the FGD participants, transcripts were used as evidences and the following have been categorized as functions of gay lingos :Gay Lingo is used as a cover up; Gay lingo is a tool to keep up with the modern trends; Gay Lingo functions as a representation of a sub-culture; Gay lingos also reflects the environment of the person; Gay lingo is recognized socially. It is used for interaction; Gay Lingo reflects our self-identity; Gay lingo presents experience; Gay lingo imparts factual information and Gay lingo controls the behaviors of others. On Circumstances That Allow Filipino Teenagers to Often Use Gay Lingos as Word Substitutes, the speakers in this conversational transcript are talking about the circumstances where they could use the gay lingo.

There are three circumstances that have been categorized in the transcript.

\subsection{On when gay students use the word substitutes}

As mentioned by the FGD participants, transcripts were used as evidences. Gay students use the word substitute when there is a need for private conversation; Gay students use word substitute when there is a need to be creative and Gay students use word substitute when they have to entertain.

\subsection{On where gay students use the word substitute}

As mentioned by the FGD participants, transcripts were used as evidences. Gay students use the word substitute everywhere, during social gatherings, public places, and meeting places.

\subsection{On why gay students use the word substitutes}

As mentioned by the FGD participants, transcripts were used as evidences. Gay students use the word substitute to hide agenda, to take care for the feelings of others, for privacy, to protect one's self from conflicts and to filter the words.

\subsection{On how do gay students use the word substitutes}

Gay students use word substitute by hiding real meanings of words, by using non-verbal cues, by changing traditional words to new words, by inserting humour and by exaggerating the meaning of the words.

\subsection{On How Gay Lingos Project Social Identity (Thematic Insights)}

The speakers in this conversational transcript were talking about how gay lingo projects their social identity. Projections of social identity had been categorized in the transcript. The use of gay lingos socially identifies them as creative individuals. Gay lingos also socially 
labelled them as closeted individuals. Gay lingos socially constructed gay men as closeted yet creative individuals. Gay Lingo reflects our self-identity.

\section{CONCLUSIONS}

The implications of the use of these gay lingos show the gays' way of integrating themselves in the society in their own terms, making serious as satirized or light and makes the language as an act of subversion.

The following were the conclusions after which findings of the study were formulated:

\section{A. On Functions of Gay Lingo}

Gay Lingos function as a cover up and a tool to keep up with the modern trends, a representation of a sub-culture, and a reflection of the environment of a person. They are used socially and are used for interaction. They reflect their self-identity, presents their experience, impart factual information, and control the behaviour of others.

B. On circumstances that allow Filipino teenagers to use gay lingo as word substitutes during conversations

The circumstances that allow Filipino teenagers to use gay lingos as word substitute are as follows: They use the word substitute in circumstances that require them to replace vulgar words so as not to hurt feelings of others, in circumstances where taboo issues such as sexual misdemeanour confronts them to isolate themselves, and on circumstances that need their satirical front/selves in public entertainment.

\section{On projection of social identity}

Projections of social identity had been categorized in the transcript. The use of gay lingos socially identifies them as creative individuals. Gay lingos also socially labelled them as closeted individuals. Gay lingos socially constructed gay men as closeted yet creative individuals. Gay Lingo as Reflection of Social Identify was anchored on the different theories related to language acquisition such as Queer Theory, Sociolinguistics and Social Identity Theory. In this study, the findings from FGD had provided directions for further understanding of the significant impact of the participation of participants. When it comes to the functions of gay lingo, the gay lingos were used as a cover up and a tool to keep up with the modern trends.On circumstances that allow Filipino teenagers to use gay lingos as word substitutes during conversations, they use the word substitute in circumstances that require them to replace vulgar words so as not to hurt feelings of others, in circumstances where taboo issues such as sexual misdemeanour confronts them to isolate themselves, and in circumstances that need their satirical front/selves in public entertainment. On projection of social identity, gay lingos socially construct gay men as closeted yet creative individuals.

Overall, the Gay Lingo As Reflection of Social Identify has proven to be meaningful venue for language instructors to be aware of the strength and weaknesses of this particular group of students when it comes to the identity that they are projecting not only in school but also in the community where they belong which would allow them to use gay lingos as word substitutes and be responsible for the kind of words that they are using during conversation. 
